\title{
"Isolated central retinal artery occlusion as a presenting manifestation of cardiac myxoma."
}

\author{
Mukesh Yadav ${ }^{1}$, Satyajit Singh ${ }^{2}$, Samdish Sethi ${ }^{1}$, Preeti Singh ${ }^{3}$, NITIN KASHYP ${ }^{4}$, and \\ Atul Kaushik ${ }^{5}$ \\ ${ }^{1}$ AIIMS Raipur \\ ${ }^{2}$ Sanjay Gandhi PGIMS \\ ${ }^{3}$ Sai Baba eye hospital \\ ${ }^{4}$ kasturba medical college \\ ${ }^{5}$ All India Institute of Medical Sciences Jodphur
}

October 23, 2020

\begin{abstract}
Background: Central retinal Artery occlusion (CRAO) is an ophthalmic emergency and the ocular analogue of the cerebral stroke. Atrial myxomas are the most common benign primary cardiac tumor. We report a case of undiagnosed left atrium (LA) myxoma who presented with sudden onset blindness in right eye due to CRAO as a sole manifestation and echocardiographic characteristics of myxoma which increases the risk of embolism. Methods: A 52-year old woman presented with history of sudden onset blindness in right eye. Fundus examination was suggestive of CRAO. Transthoracic and transesophageal echocardiogram showed a mass in LA compatible with LA myxoma. Complete surgical resection of myxoma was done although vision could not be restored to normal. Conclusion: Detailed history and complete systemic examination should be done in every patient with embolic phenomena and early neuroimaging with echocardiography using newer modality like 3D imaging should be used even in absence of electrocardiographic or auscultatory abnormalities. Key words: Myxoma, retinal artery occlusion, embolism, 3 dimensional echocardiography
\end{abstract}

Title: "Isolated central retinal artery occlusion as a presenting manifestation of cardiac myxoma."

Mukesh Yadav, DM ${ }^{1}$, Satyajit Singh, DM ${ }^{1}$, Samdish Sethi, MBBS ${ }^{2}$, Preeti Singh, MS $^{3}$, Nitin Kashyap, $\mathrm{Mch}^{4}$, Atul Kaushik, DM ${ }^{5}$,

1. Assistant Professor, Department of cardiology, AIIMS Raipur

2. Junior Resident, Department of cardiology, AIIMS Raipur

3. Consultant, Sai Baba Eye Hospital, Raipur

4. Associate Professor, Department of Cardiothoracic Surgery, AIIMS Raipur

5. Assistant Professor, Department of cardiology, AIIMS Jodhpur

\section{Corresponding author}

Dr. Mukesh Yadav

Assistant Professor, Department of cardiology, AIIMS Raipur

Email:drmukki@gmail.com

Phone: +919808953280 
Office Address: AIIMS OPD Block, Ground floor B-Block, AIIMS, Raipur, Chhattisgarh, India. PIN492099

\section{Financial disclosure}

None

\section{Declaration of conflict of interest}

None

Title: Isolated central retinal artery occlusion as a presenting manifestation of cardiac myxoma.

\section{Abstract:}

Background: Central retinal Artery occlusion (CRAO) is an ophthalmic emergency and the ocular analogue of the cerebral stroke. Atrial myxomas are the most common benign primary cardiac tumor. We report a case of undiagnosed left atrium (LA) myxoma who presented with sudden onset blindness in right eye due to $\mathrm{CRAO}$ as a sole manifestation and echocardiographic characteristics of myxoma which increases the risk of embolism.

Methods: A 52-year old woman presented with history of sudden onset blindness in right eye. Fundus examination was suggestive of CRAO. Transthoracic and transesophageal echocardiogram showed a mass in LA compatible with LA myxoma. Complete surgical resection of myxoma was done although vision could not be restored to normal.

Conclusion: Detailed history and complete systemic examination should be done in every patient with embolic phenomena and early neuroimaging with echocardiography using newer modality like 3D imaging should be used even in absence of electrocardiographic or auscultatory abnormalities.

Key words : Myxoma, retinal artery occlusion, embolism, 3 dimensional echocardiography

Introduction: Myxomas are the most common benign primary cardiac tumor in adults. Most myxomas $(>80 \%)$ are found in Left atrium (LA), although also reported in right atrium, right ventricle and left ventricle with decreasing frequency ${ }^{1}$. Incidence of cardiac myxoma peak at 40 to 60 years of age with male to female ratio of approximately $1: 3^{1}$. Cardiac myxoma may present with obstructive, embolic and constitutional symptoms including fever, weight loss, fatigue or combination of these ${ }^{1}$. We report a case of sudden onset painless loss of vision in the right eye due to central retinal artery occlusion (CRAO) as an isolated manifestation of undiagnosed LA myxoma.

Materials and methods: Written informed consent for this work to be published (including case history, images and data) was obtained from the patient for publication of this case report, including accompanying images. A 52-year-old woman presented with history of sudden onset painless loss of vision in right eye, one month back in September 2020. She was previously evaluated by an ophthalmologist at a nearby centre, diagnosed as a case of central retinal artery occlusion (CRAO) of the right eye and was being treated with corticosteroids, aspirin, and atorvastatin. On admission, her visual acuity in right eye was limited to only perception of light with inaccurate projection of rays. Visual field of right eye was defective with absent pupillary light reflex. Applanation tonometry was normal and there was no ocular movement limitation. Left eye vision was normal. Her blood pressure was $110 / 70 \mathrm{mmHg}$ and pulse rate was 68 beats per minutes. Her systemic examination was within normal limits, including normal cardiovascular system examination with no abnormal murmur, bruit or tumor plop. Fundus examination of right eye was suggestive of CRAO with whitening and opacification of the retina especially at posterior pole with a cherry red spot in the fovea (Figure 1a). Fluorescein angiography of right eye was also suggestive of CRAO with arterial phase being extremely delayed and masking of choroidal circulation due to swelling of overlying retina (Figure 1b.). Neuroimaging including diffusion weighted MRI scan showed no significant abnormalities. Her electrocardiogram showed normal sinus rhythm. Bilateral carotid artery doppler revealed no significant abnormalities. Her blood investigations including complete blood count with erythrocyte sedimentation rate (ESR), renal function test 
was within normal limits. Transthoracic echocardiogram (TTE) was done which revealed a soft gelatinous mass of $31 \times 21 \mathrm{~mm}$ size in LA, attached to atrial septum through a stalk suggestive of LA myxoma (Figure 2). Transesophageal echocardiogram (TEE) was done for morphological detailing of LA myxoma, revealed a soft gelatinous mass attached to left atrium septum by stalk and having multiple irregular fragile villous extensions from the surface of tumor (Figure $3 \mathrm{a}$. and $3 \mathrm{~b}$ ). Live 3 Dimensional (3D) echocardiographic images provided more detailing of morphological characteristics of tumor, including multiple small irregular fragile extensions from the surface of myxoma which make the patient prone for embolic phenomenon (Figure 4). To prevent recurrence of embolic event, she underwent complete surgical resection of LA myxoma via median sternotomy. The tumor was completely resected along with part of atrial septum, and the defect was repaired with autologus pericardium patch (Figure 5). Histopathological analysis of the resected mass was consistent with myxoma. The patient recovered uneventfully from cardiac surgery but unfortunately her right eye sight did not recover.

Discussion: Central retina artery occlusion is an end artery occlusion causing acute ischemia of retina, and leads to sudden onset irreversible visual impairment in the affected eye. Once the central retinal artery is occluded, the ability of the retina to recover depends on whether the offending embolus or thrombus is dislodged and more importantly retinal ischemic tolerance time ${ }^{2}$. The exact retinal ischemic tolerance time when irreversible damage occurs is not known but would be appear to be no longer than 4 hours $^{2}$. Histologically, myxomas are composed of spindle and stellate shaped cells with myxoid stroma, may also contain endothelial cells, smooth muscle cells and surrounded by mucopolysaccharide substance ${ }^{3}$. Cardiac myxoma could be asymptomatic and may be diagnosed as an incidental finding on echocardiogram. When symptomatic, it may present with features of mitral valve obstruction (54-95\%), systemic embolism (10-45\%) and constitutional symptoms such as fatigue, fever and weight loss ${ }^{4}$. In laboratory findings, there could be anemia, raised erythrocyte sedimentation rate (ESR), C-reactive protein (CRP) and gamma globulin level ${ }^{4}$. In this case, the patient was having normal laboratory investigations and normal cardiovascular system examination. Pinede et al. reported cardiac auscultation abnormalities only in $64 \%$ of patient ${ }^{4}$. So absence of auscultatory abnormalities does not rule out cardiac myxomas, as in our case. Vascular disturbance in the eye due to cardiac myxoma are rare, however embolism in ophthalmic circulation due to cardiac tumor has been reported in literature ${ }^{5}$. Acebo et al. previously reported the morphological features of myxomas which were associated with embolic phenomena. Villous or papillary forms of myxomas with fragile extension have a tendency to fragment spontaneously and associated with embolic phenomena ${ }^{6}$. In our case also the myxomas was having soft gelatinous consistency with multiple fragile villous extensions, which caused the retinal artery embolism as a primary manifestation. Echocardiography is the primary diagnostic imaging modality for intracardiac tumors. Beside transthoracic and transesophageal echo, 3D echocardiogram adds incremental value to the morphological assessment of myxomas and correlates very well with the surgical and histopathological findings, as in our case. The tumors with morphological features associated embolic phenomena should be intervened on urgent basis. Yu et al. reported a 43 year old woman with retinal artery occlusion with syncope caused by atrial myxoma, rapid diagnosis and exact treatment of myxomas improved patient's visual capacity ${ }^{7}$. But in our case, patient presented late to us for the cardiac evaluation as a part of diagnostic work-up of CRAO and vision of affected eye could not be restored, although complete resection of myxomas curtailed the future risk of embolic phenomena. Lifelong follow-up is needed in these cases as myxomas have some tendency to recur with rate of $5-14 \%$. The time to recurrence varied from 0.5 to 6.5 years in different series ${ }^{1,4}$.

Conclusion: In summary, we report this case of isolated retinal artery occlusion as a presenting manifestation of undiagnosed LA Myxoma. Ophthalmologist should consider the possibility of myxomas in patient with sudden loss of visual acuity, as timely management is essential for better outcome and prognosis in these patients. The detailed medical history with systemic examination is essential in all patients and normal cardiac auscultation does not rule out cardiac pathology. We also recommend to always look for high risk morphological features in cardiac myxomas for embolism with use of less invasive and newer modalities like 3D Echocardiography which provides better morphological characterization.

\section{Conflict of interest}


All the authors declare that there is no conflict of interest.

\section{Financial disclosure}

None

\section{Author Contributions}

Concept/design: Mukesh yadav, Data collection, analysis and interpretation: Satyjit singh, Preeti singh, Samdish sethi, Mukesh yadav, Drafting the article: Nitin kashyap, Mukesh yadav, Atul kaushik Critical review of the manuscript and approval of article: all author equally contributed.

\section{Data availability statement}

The authors declare that the data supporting the finding of this study are available within the article and its supplementary information files.

\section{References:}

1. Ekmektzoglou KA, Samelis GF, Xanthos T. Heart and tumors: location, metastasis, clinical manifestations, diagnostic approaches and therapeutic considerations. J Cardiovasc Med (Hagerstown). 2008 Aug;9(8):769-77.

2. Hayreh SS, Zimmerman MB, Kimura A, Sanon A. Central retinal artery occlusion. Retinal survival time. Exp Eye Res. 2004 Mar;78(3):723-36.

3. McManus, B., "Primary tumors of the heart", In Bonow, R.O., Mann, D.L., Zipes, D.P., Libby, P. (eds) Braunwald's Heart Disease, 9th ed, Philadelphia, Elsevier Saunders, 2011, 1638-1650.

4. Pinede L, Duhaut P, Loire R. Clinical presentation of left atrial cardiac myxoma. A series of 112 consecutive cases. Medicine (Baltimore). 2001 May;80(3):159-72.

5. Schmidt D, Hetzel A, Geibel-Zehender A: Retinal arterial occlusion due to embolism of suspected cardiac tumors - report on two patients and review of the topic. Eur J Med Res 2005, 10(7):296-304.

6. Acebo E, Val-Bernal JF, Gómez-Román JJ, Revuelta JM. Clinicopathologic study and DNA analysis of 37 cardiac myxomas: a 28-year experience. Chest. 2003 May;123(5):1379-85.

7. Yu, Y., Zhu, Y., Dong, A. et al. Retinal artery occlusion as the manifestation of left atrial myxoma: a case report. BMC Ophthalmol 14, 164 (2014).

\section{Figure legends:}

Figure 1: (1a) Colour Fundus photograph of right eye with acute central retinal artery occlusion (CRAO) showing opacification of retina especially at posterior pole with cherry red spot at the center. (1b) Corresponding fluorescein angiography of right eye showing masking of choroidal circulation due to swelling of overlying retina and extremely delayed arterial phase with incomplete filling of the arteries of right eye even at 19 seconds as compared to the arteries of left eye.

Figure 2: Trans-thoracic echocardiogram in apical 4 chamber view in showing pedunculated hyperechoic mass (white arrow) in left atrium suggestive of left atrium myxoma attached to atrial septum.

Figure 3: (3a) Trans-esophageal echocardiogram (TEE) with Modified 3 chamber view showing left atrium myxoma with soft gelatinous consistency. (3b) TEE showing multiple irregular villous extensions from myxoma surface.

Figure 4: 3 Dimensional (3D) echocardiogram in apical 4- chamber view showing Left atrium myxoma attached to atrial septum and having multiple irregular villous extensions (arrow) from myxoma surface.

Figure 5: Resection of left atrium myxoma (arrow), Myxoma showing soft gelatinous consistency with irregular villous extensions from surface. 

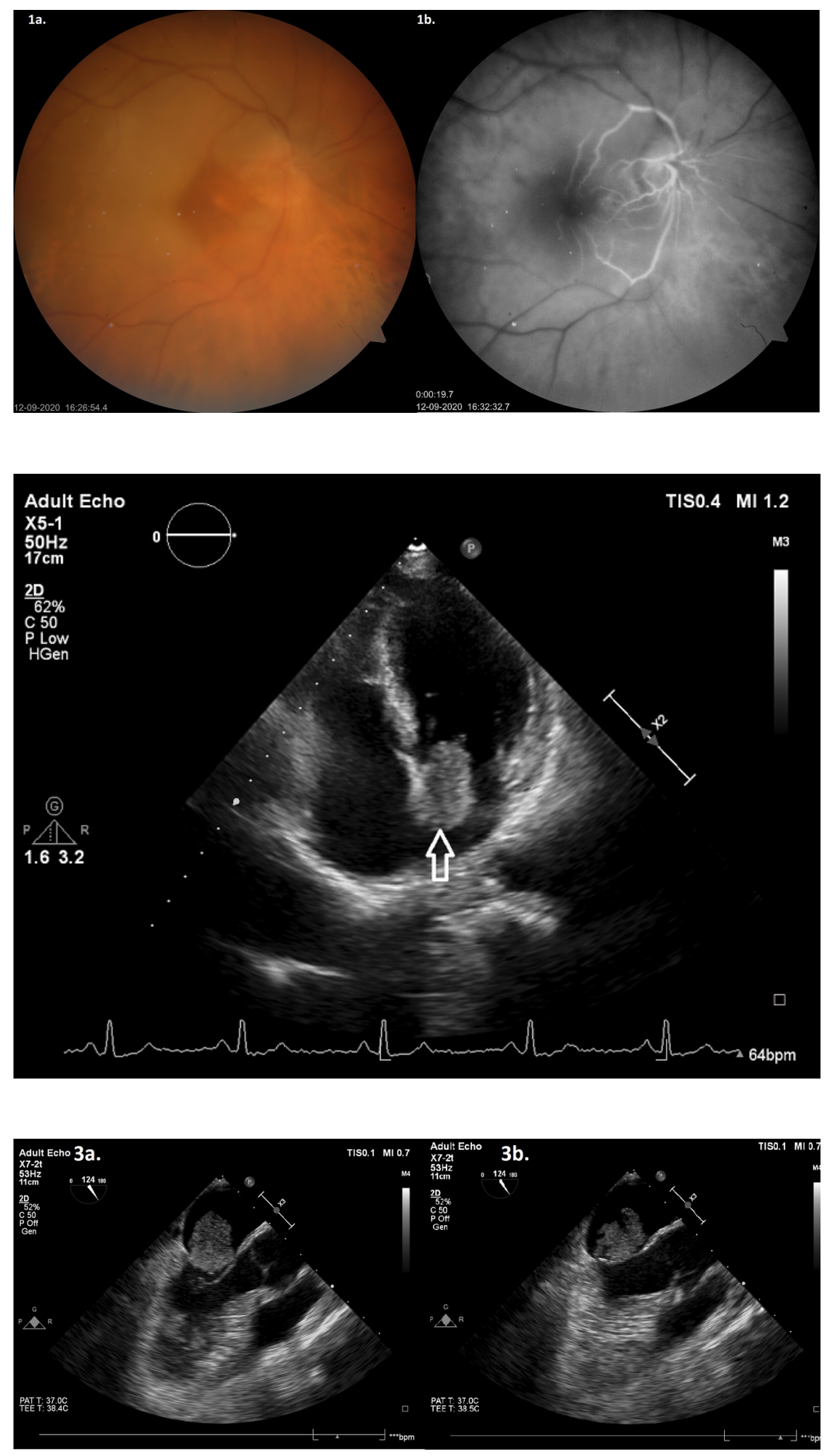

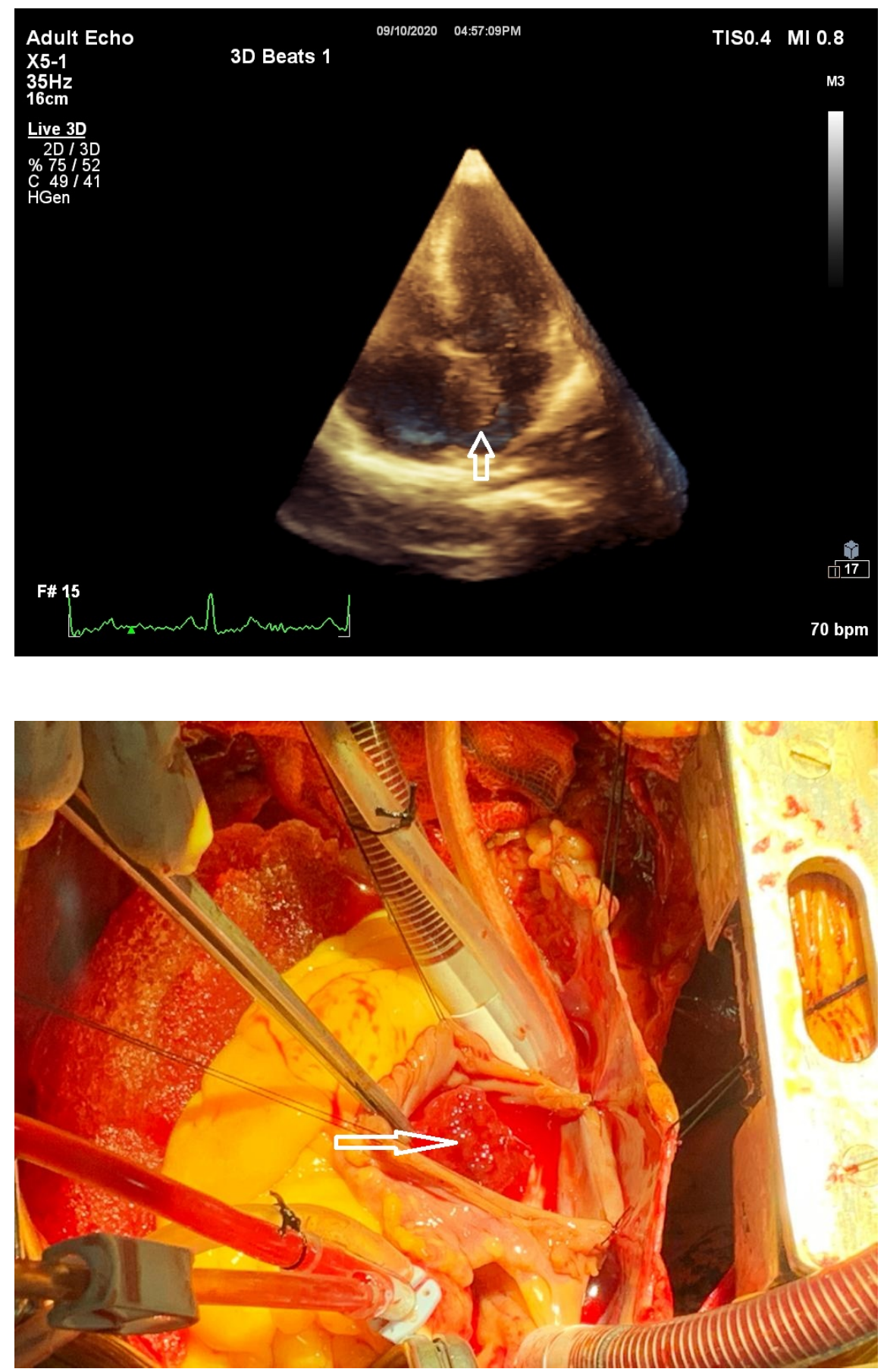